\title{
MRI-based radiomics of rectal cancer: preoperative assessment of the pathological features
}

\author{
Xiaolu Ma ${ }^{1}$, Fu Shen ${ }^{1 *}$, Yan Jia ${ }^{2}$, Yuwei Xia ${ }^{2}$, Qihua $\mathrm{Li}^{2}$ and Jianping Lu
}

\begin{abstract}
Background: This study aimed to evaluate the significance of MRI-based radiomics model derived from highresolution T2-weighted images (T2WIs) in predicting tumor pathological features of rectal cancer.

Methods: A total of 152 patients with rectal cancer who underwent surgery without any neoadjuvant therapy between March 2017 and September 2018 were included retrospectively. The patients were scanned using a 3-T magnetic resonance imaging, and high-resolution T2Wls were obtained. Lesions were delineated, and 1029 radiomics features were extracted. Least absolute shrinkage and selection operator was used to select features, and multilayer perceptron (MLP), logistic regression (LR), support vector machine (SVM), decision tree (DT), random forest (RF), and K-nearest neighbor (KNN) were trained using fivefold cross-validation to build a prediction model. The diagnostic performance of the prediction models was assessed using the receiver operating characteristic curves.
\end{abstract}

Results: A total of 1029 features were extracted, and 15,11, and 11 features were selected to predict the degree of differentiation, $\mathrm{T}$ stage, and $\mathrm{N}$ stage, respectively. The best performance of the radiomics model for the degree of differentiation, T stage, and N stage was obtained by SVM [area under the curve (AUC), 0.862; 95\% confidence interval (Cl), 0.750-0.967; sensitivity, 83.3\%; specificity, 85.0\%], MLP (AUC, 0.809; 95\% Cl, 0.690-0.905; sensitivity, 76.2\%; specificity, 74.1\%), and RF (AUC, 0.746; 95\% Cl, 0.622-0.872; sensitivity, 79.3\%; specificity, 72.2\%).

Conclusion: This study demonstrated that the high-resolution T2WI-based radiomics model could serve as pretreatment biomarkers in predicting pathological features of rectal cancer.

Keywords: Histological grade, Magnetic resonance imaging, N stage, Radiomics, Rectal cancer, T stage

\section{Background}

Colorectal cancer $(\mathrm{CRC})$ is the third most common malignant tumor worldwide [1]. According to the latest data, reported by the Cancer Statistics of China in 2015, CRC ranks fifth in morbidity and mortality [2]. Among all the patients with CRC, rectal cancer accounts for 30$35 \%$, which are generally adenocarcinomas. The optimal therapy program selection is a multifarious course for patients with rectal cancer [3, 4], and an accurate preoperative stage is an essential step for guiding treatment decisions, including surgery or neoadjuvant chemoradiotherapy (nCRT). Surgical excision is regarded as the

\footnotetext{
* Correspondence: ssff_53@163.com

'Department of Radiology, Changhai Hospital of Shanghai, Shanghai, China Full list of author information is available at the end of the article
}

standard treatment strategy for early rectal cancer (T1-2 and N0), and the treatment for locally advanced (T3-4 and/or N1) rectal cancer is nCRT followed by total mesorectal excision surgery [3]. Generally, the pathological type, degree of differentiation, depth of infiltration, and presence or absence of regional lymph node metastasis reflect the degree of tumor invasiveness and predict the prognosis of a tumor [3]. Therefore, a deeper understanding of tumor pathological features has a critical value in formulating the clinical treatment plan and predicting the prognosis. Moreover, high-resolution magnetic resonance imaging (MRI) has a pivotal role in the pretreatment assessment of rectal cancer because the high-resolution T2-weighted images (T2WIs) offer better diagnostic performance in the staging of rectal cancer [3].

(c) The Author(s). 2019 Open Access This article is distributed under the terms of the Creative Commons Attribution 4.0 International License (http://creativecommons.org/licenses/by/4.0/), which permits unrestricted use, distribution, and reproduction in any medium, provided you give appropriate credit to the original author(s) and the source, provide a link to the Creative Commons license, and indicate if changes were made. The Creative Commons Public Domain Dedication waiver (http://creativecommons.org/publicdomain/zero/1.0/) applies to the data made available in this article, unless otherwise stated. 
Recently, radiomics analysis was developed and validated as an advanced tool in assessing tumor heterogeneity. Radiomics is a noninvasive method that involves highquality image acquisition, VOIs segmentation, highthroughput quantitative feature extraction, high-dimension feature reduction, and diagnostic, prognostic, or predictive model establishment. The radiomics model, which makes use of the medical images and clinical data, has a potential in clinical decision-making [5]. Radiomics has been used to evaluate several kinds of tumors in previous studies and is being increasingly implemented [5-9]. MRI-based radiomics model has been employed in distinguishing cancer from benign tissue and reflecting the histological characteristics of rectal cancer [10-13]. Therefore, the purpose of the present study was to investigate the significance of an MRI-based radiomics model derived from high-resolution T2WI in identifying specific pathological features of rectal cancer and build a set of prediction radiomics models.

\section{Methods \\ Participants}

This retrospective study was approved by the local institutional (Committee on Ethics of Biomedicine, Second Military Medical University) review board, and written informed consent was waived for each patient. Between March 2017 and September 2018, 182 consecutive patients with rectal lesions identified by colonoscopy with no previous treatment were involved in this study. All patients underwent rectal MRI examination and postoperative pathological test. The exclusion criteria were as follows: chemotherapy or radiotherapy before and after MRI $(n=20)$, poor image quality $(n=6)$, and distant metastases $(n=4)$. Therefore, 152 patients were included in the final analysis.

\section{Magnetic resonance imaging}

All patients were scanned on a $3 \mathrm{~T}$ MRI (MAGNETOM Skyra, Siemens Healthcare, Erlangen, Germany) using an 18-channel pelvic phased-array coil. Every patient fasted for $4 \mathrm{~h}$ prior to the scan. Transversal high-resolution T2- weighted turbo spin echo images were acquired with the following parameters: $\mathrm{TR} / \mathrm{TE}=4000 / 108 \mathrm{~ms}, \quad \mathrm{FOV}=$ $180 \times 180 \mathrm{~mm}^{2}$, matrix $=320 \times 320$, slice thickness $=3$ $\mathrm{mm}$, gap $=0 \mathrm{~mm}$, acceleration factor $=3$, echo train length $=16$, and acquisition time $=4 \mathrm{~min} 10 \mathrm{~s}$. All patients underwent surgery at a time interval of $8.9 \pm 5.8$ (range, 2-28) days after the MRI examination.

\section{Pathological evaluation}

The tissue sections were subjected to hematoxylin and eosin staining. All lymph nodes in the mesorectum were retrieved from the surgical specimens to ensure that at least 12 lymph nodes per patient were collected. The final histopathological reports detailed the tumor TN staging, histological grade, and circumferential resection margin (CRM). All TN statuses were determined according to the American Joint Committee on Cancer staging system, eighth edition $[14,15]$. The patients were divided into two groups according to different pathological criteria. Histological grade: high-to-moderate and poor differentiation; T stage: T1-2 and T3-4 stages; and $\mathrm{N}$ stage: N0 and N1-2 stages.

\section{Feature selection}

The radiomics features were extracted from the VOIs as confirmed by a radiologist (with 8 years of experience in radiology) on high-resolution T2WI using a radiomics analysis platform [Radcloud, Huiying Medical Technology (Beijing, China) Co., Ltd.] (Fig. 1). 1029 high-throughput data features based on feature classes and filter classes were automatically extracted from the platform. The platform feature extraction is based on the "pyradiomics" package in Python (version 2.1.2, https://pyradiomics. readthedocs.io/).

To minimize the MRI intensity variations, we normalized the intensity of the image using the following formula (where $x$ indicates the original intensity; $f(x)$ indicates the normalized intensity; $\mu$ refers to the mean value; $\sigma$ indicates the variance; $s$ is an optional scaling, by default, it is set to 1 ).
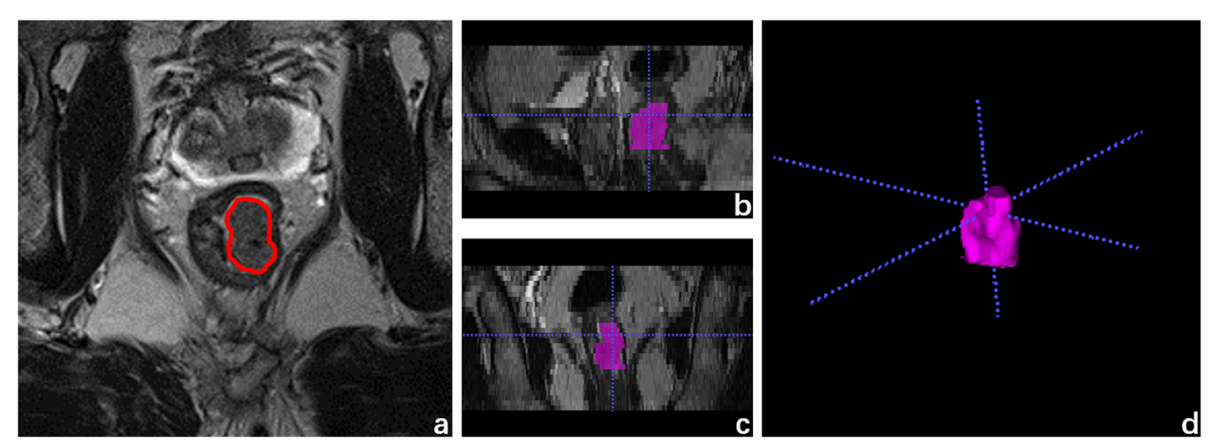

Fig. 1 Example image for rectal cancer contouring. a The outline of ROI on one slice of axial T2-weighted MR image. b Sagittal reconstruction. c Coronal reconstruction. d Volume rendering 


$$
f(x)=\frac{s\left(x-\mu_{x}\right)}{\sigma_{x}}
$$

First, to guarantee image feature robustness, the basis of an intraclass correlation of 0.6 was set for test-retest analysis. Then, the robust features were selected by the least absolute shrinkage and selection operator (LASSO) method to best predict the classification performance. In the LASSO method, leave-one-out cross-validation was used to select the optimal regularization parameter alpha, as the average of mean square error of each patient was the smallest. With the optimal alpha, features having nonzero coefficient in LASSO were reserved.

\section{Prediction model analysis}

The machine learning is based on the "scikit-learn" package in Python (version 0.21.3, https://scikit-learn. org/stable/). The original collection was divided into a training set (70\%) and a test set (30\%) randomly. Moreover, to lower the imbalance impact of samples distribution of the degree of histological grade and $\mathrm{N}$ stage, the synthetic minority oversampling technique algorithm was used in the training set. The multilayer perceptron (MLP), logistic regression (LR), support vector machine (SVM), decision tree (DT), random forest (RF), and Knearest neighbor $(\mathrm{KNN})$ classifiers were trained (the parameters of the six classifiers are shown in Table 1) using fivefold cross-validation to build a prediction model. Moreover, the independent test set was used to test the performance of the model. The experiment used the mean model as the final model for the test set. The performance of models for the statistically significant pathological features was assessed using sensitivity, specificity, and area under the receiver operating characteristic (ROC) curve (AUC). $P$ value $<0.05$ was considered statistically significant.

\section{Results}

\section{Patient demographics}

Among the 152 patients with rectal cancer, 94 were male and 58 were female, with a mean age of $58.9 \pm$ 8.3 years (range 24-78). The pathological features of rectal cancer are presented in Table 2. None of them had positive CRM.

\section{Diagnostic performance of radiomics}

A total of 1029 features were extracted from preoperative high-resolution T2WI, can be classified into three categories as follows: I. The characteristics of intensity statistics, such as peak value, mean value, and variance, which are used to quantitatively describe the distribution of voxel intensity in MR images; II. Shape features, such as volume, surface area, and spherical value, which reflect the three-dimensional characteristics of the shape and size of the outlined area; and III. texture features, including the gray-level co-occurrence matrix, gray-level run length matrix, and gray-level size zone matrix, which can quantify the heterogeneity of the selected region. Additionally, Laplace-Gauss filtering, exponential, logarithmic, square, square root, and wavelet filters can be used to calculate image intensity and texture features. Wavelet filters used included wavelet-LHL, wavelet-LHH, wavelet-HLL, wavelet$\mathrm{LLH}$, wavelet-HLH, wavelet-HHHH, wavelet-HHL, and wavelet-LLL. Then 15,11 , and 11 characteristic features related to the degree of differentiation, $\mathrm{T}$ stage, and $\mathrm{N}$ stage, respectively, were obtained (Table 3). Radiomics features were selected for subsequent prediction model building, the cutoff value was selected according to the Youden index to determine the corresponding sensitivity and specificity. The AUC was used to assess the predictive ability of the model, and the selection results are presented in Tables 4 and 5.

For the degree of differentiation, the SVM classifier provided the best discrimination capability for the prediction model with an AUC of 0.862 (95\% CI, 0.7500.967; sensitivity, 83.3\%; specificity, $85.0 \%$ ). As for the T stage, the MLP classifier provided the best discrimination capability with an AUC of 0.809 (95\% CI, 0.6900.905; sensitivity, 76.2\%; specificity, $74.1 \%)$. Moreover, the RF classifier showed a good diagnostic performance for the N stage with an AUC of 0.746 (95\% CI, 0.6220.872; sensitivity, 79.3\%; specificity, 72.2\%) (Fig. 2).

\section{Discussion}

This study indicated that the high-resolution T2WIbased radiomics machine learning model could not only differentiate pathological differentiation and $\mathrm{T}$ stage but also exhibited good diagnostic performance for $\mathrm{N}$ stage.

Recent studies have shown that radiomics is important in identifying tumor heterogeneity in several kinds of

Table 1 Supplemental data (parameters)

\begin{tabular}{|c|c|c|c|}
\hline Model & Degree of Differentiation & T stage & $\mathrm{N}$ stage \\
\hline MLP & hidden_layer_sizes = (30) & hidden_layer_sizes = $(30)$ & hidden_layer_sizes = (30) \\
\hline$L R$ & penalty = 'I2', solver = 'liblinear' & penalty = 'I2', solver = 'liblinear' & penalty $=$ ' $\mid 2^{\prime}$, solver = 'liblinear' \\
\hline SVM & kernel = 'rbf', probability = True & kernel = 'Poly', probability = True & kernel = 'rbf', probability = True \\
\hline DT & criterion = 'gini' & criterion = 'gini' & criterion = 'gini' \\
\hline RF & n_estimators $=15$ & n_estimators $=15$ & n_estimators $=15$ \\
\hline KNN & n_neighbors $=5$ & n_neighbors $=5$ & n_neighbors $=5$ \\
\hline
\end{tabular}


Table 2 Pathological characteristics of the patients

\begin{tabular}{|c|c|c|c|}
\hline pathological characteristics & $\begin{array}{l}\text { Total } \\
\text { n percentile (\%) }\end{array}$ & $\begin{array}{l}\text { Training data (70\%) } \\
\text { n percentile (\%) }\end{array}$ & $\begin{array}{l}\text { Test data (30\%) } \\
\text { n percentile (\%) }\end{array}$ \\
\hline \multicolumn{4}{|l|}{ Gender } \\
\hline Male & $94(61.8)$ & $63(59.4)$ & $31(67.4)$ \\
\hline Female & $58(38.2)$ & $43(40.6)$ & $15(32.6)$ \\
\hline \multicolumn{4}{|l|}{ Age (years) } \\
\hline Mean & $58.9 \pm 8.3$ & $52.3 \pm 10$ & $58.9 \pm 8.0$ \\
\hline Range & $24-78$ & $24-77$ & $25-78$ \\
\hline \multicolumn{4}{|l|}{ Histological type } \\
\hline Adenocarcinoma & $131(86.2)$ & $91(85.8)$ & $40(87.0)$ \\
\hline Mucinous adenocarcinoma & $15(9.9)$ & $11(10.4)$ & $4(8.7)$ \\
\hline Signet ring cell carcinoma & $6(3.9)$ & $4(3.8)$ & $2(4.3)$ \\
\hline \multicolumn{4}{|l|}{ Pathologic differentiation } \\
\hline High & $20(13.2)$ & $14(13.2)$ & $6(13.0)$ \\
\hline Moderate & $112(73.7)$ & $78(73.6)$ & $34(73.9)$ \\
\hline Poor & $20(13.2)$ & $14(13.2)$ & $6(13.0)$ \\
\hline \multicolumn{4}{|l|}{ T stage } \\
\hline T1 & $22(14.5)$ & $15(14.2)$ & $7(15.2)$ \\
\hline $\mathrm{T} 2$ & $44(28.9)$ & $28(26.4)$ & $16(34.8)$ \\
\hline T3 & $74(48.7)$ & $53(50.0)$ & $21(45.7)$ \\
\hline T4 & $12(7.9)$ & $10(9.4)$ & $2(4.3)$ \\
\hline \multicolumn{4}{|l|}{ N stage } \\
\hline NO & $94(61.9)$ & $67(63.2)$ & $27(58.7)$ \\
\hline $\mathrm{T} 1$ & $37(24.3)$ & $27(25.5)$ & $10(21.7)$ \\
\hline $\mathrm{T} 2$ & $21(13.8)$ & $12(11.3)$ & $9(19.6)$ \\
\hline
\end{tabular}

Table 3 Radiomics features

\begin{tabular}{|c|c|c|c|}
\hline No & Degree of differentiation & T stage & N stage \\
\hline 1 & original_firstorder_Minimum & original_shape_Size & WaveletHLH_firstorder_Medianvalue \\
\hline 2 & original_firstorder_Entropy & WaveletLLH_firstorder_Medianvalue & WaveletHLL_glrlm_SRLGE \\
\hline 3 & original_shape_Compactness & WaveletLHH_firstorder_Meanvalue & WaveletHHL_firstorder_Energy \\
\hline 4 & original_glrlm_RLV & WaveletLHH_firstorder_Uniformity & WaveletLLH_firstorder_Medianvalue \\
\hline 5 & WaveletLLH_firstorder_Skewness & WaveletHHL_firstorder_Medianvalue & WaveletHHH_glszm_LGZE \\
\hline 6 & WaveletLLH_firstorder_Uniformity & WaveletLLL_glszm_SZE & WaveletLLL_glrlm_LRHGE \\
\hline 7 & WaveletHLH_firstorder_Kurtosis & WaveletLLL_glszm_ZSN & WaveletHHL_firstorder_Skewness \\
\hline 8 & WaveletLHL_glszm_LGZE & WaveletLLL_ngtdm_Coarseness & WaveletLLL_glcm_cshad \\
\hline 9 & WaveletLLL_glrlm_LRHGE & WaveletHLH_glcm_inf1h & WaveletLLL_glrlm_HGRE \\
\hline 10 & WaveletHHH_glrlm_RLV & WaveletHHL_glcm_senth & WaveletHLL_ngtdm_Coarseness \\
\hline 11 & WaveletHHH_glszm_LGZE & WaveletHHL_glrlm_LRHGE & WaveletHLL_glcm_inf1h \\
\hline 12 & WaveletHHL_glcm_inf2h & & \\
\hline 13 & WaveletHHH_glcm_cprom & & \\
\hline 14 & WaveletHHH_glcm_corrm & & \\
\hline 15 & WaveletLHH_glrlm_GLV & & \\
\hline
\end{tabular}


Table 4 Training set

\begin{tabular}{|c|c|c|c|c|c|c|}
\hline pathological features & model & mean AUC & std & sensitivity & specificity & Youden index \\
\hline \multirow[t]{6}{*}{ degree of differentiation } & MLP & 0.942 & 0.041 & 0.871 & 0.978 & 0.849 \\
\hline & $L R$ & 0.874 & 0.052 & 0.806 & 0.903 & 0.709 \\
\hline & SVM & 0.871 & 0.037 & 0.806 & 0.892 & 0.698 \\
\hline & DT & 0.892 & 0.040 & 1.0 & 1.0 & 1.0 \\
\hline & RF & 0.983 & 0.020 & 1.0 & 1.0 & 1.0 \\
\hline & KNN & 0.933 & 0.062 & 0.978 & 0.860 & 0.838 \\
\hline \multirow[t]{6}{*}{ T stage } & MLP & 0.824 & 0.087 & 0.804 & 0.900 & 0.704 \\
\hline & $L R$ & 0.792 & 0.083 & 0.826 & 0.733 & 0.559 \\
\hline & SVM & 0.764 & 0.083 & 0.913 & 0.783 & 0.696 \\
\hline & DT & 0.722 & 0.060 & 1.0 & 1.0 & 1.0 \\
\hline & $\mathrm{RF}$ & 0.713 & 0.031 & 1.0 & 0.983 & 0.983 \\
\hline & KNN & 0.712 & 0.081 & 0.956 & 0.600 & 0.556 \\
\hline \multirow[t]{6}{*}{ N stage } & MLP & 0.694 & 0.122 & 0.861 & 0.677 & 0.538 \\
\hline & $L R$ & 0.651 & 0.089 & 0.831 & 0.492 & 0.323 \\
\hline & SVM & 0.684 & 0.143 & 0.831 & 0.738 & 0.569 \\
\hline & DT & 0.713 & 0.060 & 1.0 & 1.0 & 1.0 \\
\hline & $\mathrm{RF}$ & 0.794 & 0.100 & 1.0 & 0.954 & 0.954 \\
\hline & KNN & 0.663 & 0.060 & 1.0 & 1.0 & 1.0 \\
\hline
\end{tabular}

tumors [5-9], which may serve as a complementary tool for the preoperative tumor staging in rectal cancer [10-13]. The patients with rectal cancer required a comprehensive staging evaluation for guiding decisions regarding choice of treatment with an aim to avoid undertreatment and minimize overtreatment. Therefore, high-resolution T2WIs were used to explore the significance of MRI-based radiomics model in the preoperative diagnosis of rectal cancer in the present study.

Previous studies have shown by NCCN, degree of differentiation, $\mathrm{T}$ stage, and $\mathrm{N}$ stage are powerful prognostic factors for patients with rectal cancer [3]. Several

Table $\mathbf{5}$ Test set

\begin{tabular}{|c|c|c|c|c|c|c|}
\hline pathological features & model & AUC & $95 \% \mathrm{Cl}$ & sensitivity & specificity & Youden index \\
\hline \multirow[t]{6}{*}{ degree of differentiation } & MLP & 0.825 & $0.659-0.967$ & 0.833 & 0.750 & 0.583 \\
\hline & LR & 0.808 & $0.649-0.946$ & 0.833 & 0.725 & 0.558 \\
\hline & SVM & 0.862 & $0.750-0.967$ & 0.833 & 0.850 & 0.683 \\
\hline & DT & 0.854 & $0.700-0.963$ & 0.833 & 0.875 & 0.708 \\
\hline & RF & 0.858 & $0.735-0.964$ & 0.833 & 0.750 & 0.583 \\
\hline & KNN & 0.692 & $0.519-0.844$ & 0.833 & 0.450 & 0.283 \\
\hline \multirow[t]{6}{*}{ T stage } & MLP & 0.809 & $0.690-0.905$ & 0.762 & 0.741 & 0.503 \\
\hline & LR & 0.762 & $0.633-0.873$ & 0.714 & 0.630 & 0.344 \\
\hline & SVM & 0.753 & $0.623-0.857$ & 0.667 & 0.630 & 0.297 \\
\hline & DT & 0.667 & $0.543-0.783$ & 0.667 & 0.667 & 0.334 \\
\hline & RF & 0.727 & $0.591-0.843$ & 0.714 & 0.704 & 0.418 \\
\hline & KNN & 0.720 & $0.586-0.830$ & 0.809 & 0.407 & 0.216 \\
\hline \multirow[t]{6}{*}{ N stage } & MLP & 0.667 & $0.531-0.799$ & 0.690 & 0.722 & 0.412 \\
\hline & LR & 0.437 & $0.294-0.575$ & 0.448 & 0.444 & -0.108 \\
\hline & SVM & 0.592 & $0.435-0.736$ & 0.552 & 0.500 & 0.052 \\
\hline & DT & 0.723 & $0.599-0.832$ & 0.724 & 0.722 & 0.446 \\
\hline & RF & 0.746 & $0.622-0.872$ & 0.793 & 0.722 & 0.515 \\
\hline & KNN & 0.560 & $0.428-0.69$ & 0.621 & 0.500 & 0.121 \\
\hline
\end{tabular}



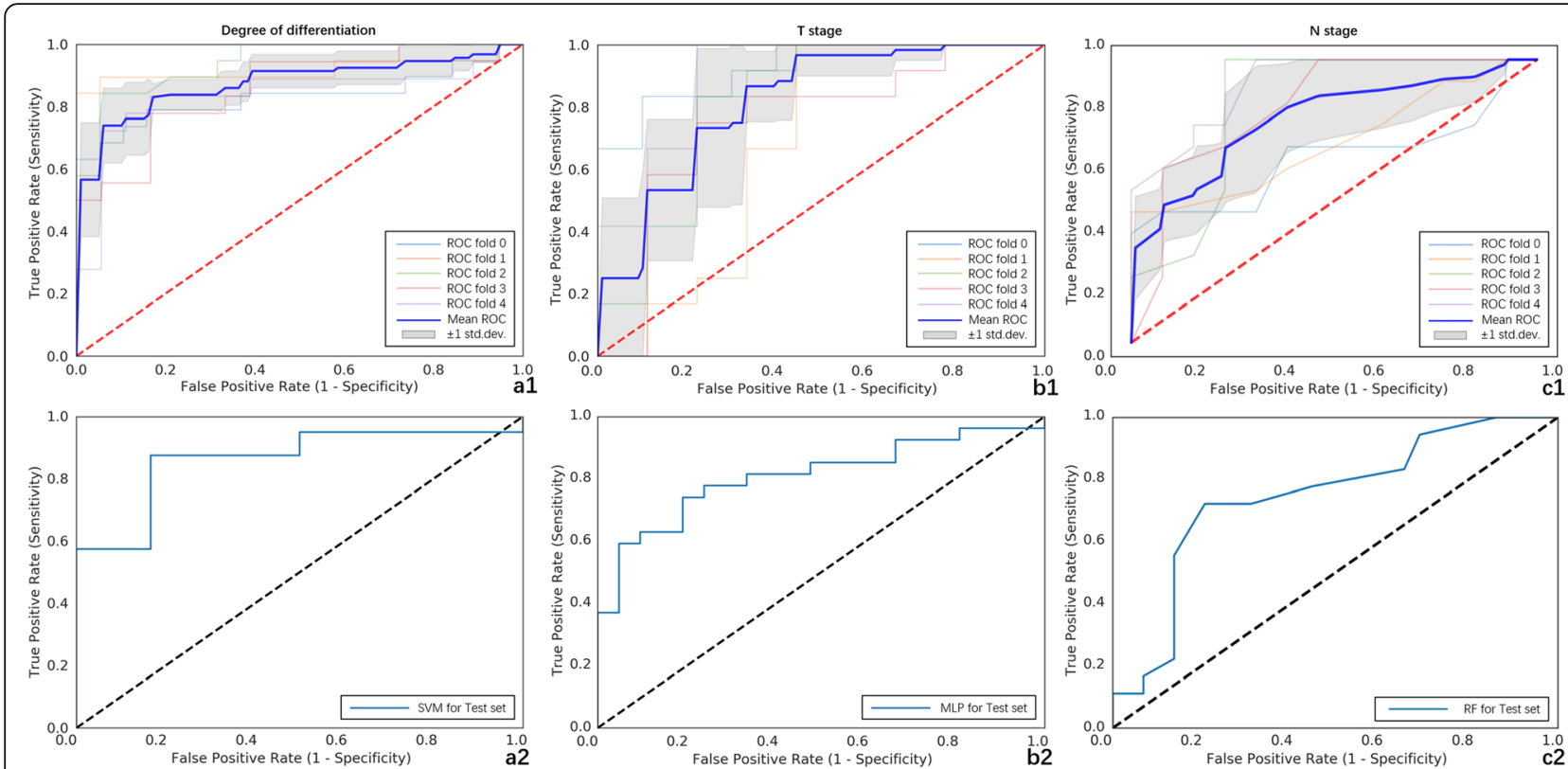

Fig. 2 Receiver operating characteristic (ROC) curves of the prediction model for the statistically significant prognostic factors. ROC curves of SVM classifier for pathological differentiation: (a1) training set (AUC, 0.871; std., 0.037; sensitivity, 80.6\%; specificity, 89.2\%); (a2) test set (AUC, 0.862; 95\% Cl, 0.750-0.967; sensitivity, 83.3\%; specificity, 85.0\%). ROC curves of MLP classifier for T stage: (b1) training set (AUC, 0.824; std., 0.087; sensitivity, 80.4\%; specificity, 90.0\%); (b2) test set (AUC, 0.809; 95\% Cl, 0.690-0.905; sensitivity, 76.2\%; specificity, $74.1 \%$ ). ROC curves of RF classifier for N stage: (c1) training set (AUC, 0.794; std., 0.100; sensitivity, 100.0\%; specificity, 95.4\%); (c2) test set (AUC, 0.746; 95\% Cl, 0.622-0.872; sensitivity, 79.3\%; specificity, 72.2\%)

studies showed a statistically significant correlation between the apparent diffusion coefficient value, derived from diffusion-weighted images, and tumor differentiation grade $[16,17]$; however, some studies showed a contradictory result $[18,19]$. In this study, radiomics and tumor differentiation grade showed a statistically significant correlation. The ROC curves of SVM classifier showed an AUC of 0.862 (test set), suggesting that the SVM model can be used to distinguish poorly differentiated lesions from highly/ moderately differentiated lesions.

Although high-resolution MRI is recommended for the $\mathrm{T}$ staging of patients with rectal cancer, the accuracy of staging is still unsatisfactory. Some studies demonstrated differences in results that ranged from 44 to $100 \%$ [20, 21]. Stage T2 lesions could be differentiated from T3 lesions by identifying a smooth outer tumor border within the rectal wall, with no invasion into the fat surrounding the rectum. The difficulty in differentiating tumor infiltration from fibrosis, which is due to inflammation and blood vessel invasion, limited the ability to distinguish stage $\mathrm{T} 2$ tumors from early-stage T3 tumors [15]. In this study, the ROC curves of MLP classifier showed an AUC of 0.809 (test set), suggesting that the MRI-based radiomics model can be used to distinguish T3-4 lesions from T1-2 lesions. These results could be explained by the fact that higher $\mathrm{T}$-stage tumors showed greater heterogeneity of cell morphology and histology, higher cell density, and smaller interstitium.
Accurate preoperative diagnosis of lymph node metastasis is another important factor for treatment selection. Although the accuracy of $\mathrm{T}$ staging is considerably high, the prediction of $\mathrm{N}$ staging remains difficult [22]. Using morphological criteria only does not improve the prediction accuracy of lymph node metastasis in rectal cancer [10]. This limitation is aggravated by the lack of consensus on appropriate criteria to assess lymph node involvement [20]. The reported accuracy of routine MRI for lymph node staging varied widely, ranging from 43 to $85 \%$ [23], suggesting that the MRI criteria for detecting lymph node metastasis are not reliable. However, the ROC curves of RF classifier showed an AUC of 0.746 (test set), which was partially consistent with the results of Huang's study [24]. The study found radiomics signatures and other risk factors could conveniently facilitate the individualized preoperative prediction of lymph node metastasis in patients with CRC. Therefore, the RF model might reflect the aggressiveness of particular tumor tissue.

This study had several limitations. First, it was a retrospective study prone to selection bias, and the exclusion of patients with distant metastases limited its application. Hence, more patients should be included to validate the results. Second, due to the relatively small sample size, some lesions were nonuniformly distributed. Further studies are needed to broaden the application of 
radiomics for these lesions. Finally, radiomics is a recent imaging modality; the MRI scanning parameters and machine learning models are not yet standardized. Large prospective multicenter trials are necessary to fully evaluate the role of radiomics in the pathological features of rectal cancer.

\section{Conclusions}

In conclusion, this study demonstrated that the highresolution T2WI-based radiomics showed good classification performance related to tumor pathological features in patients with rectal cancer. Thus, radiomics may serve as a good alternative for evaluating the pathological features of rectal cancer and can add a further dimension to the predictive power of imaging.

\section{Abbreviations}

ADC: Apparent diffusion coefficient; AUC: Area under the curve;

CRM: Circumferential resection margin; DWl: Diffusion-weighted imaging;

FOV: Field of view; LASSO: Least absolute shrinkage and selection operator: MRI: Magnetic resonance imaging; NCCN: National comprehensive cancer network; RC: Rectal cancer; ROC: Receiver operating characteristic; TME: Total mesorectal excision; TR/TE: Repetition time/echo time; VOI: Volume of interest

\section{Acknowledgements}

Not applicable

\section{Authors' contributions}

$J \mathrm{~L}$ and $\mathrm{FS}$ conceived of the present idea. $Y J$ analyzed and interpreted the patient data regarding the radiomics features. XM was a major contributor in writing the manuscript. All authors read and approved the final manuscript.

\section{Funding}

Youth Initiative Fund of Second Military Medical University (Grant Number:2018QN05). The role of the funder(s): conceived of the present idea in the design of the study.

\section{Availability of data and materials}

Not applicable

\section{Ethics approval and consent to participate}

This retrospective study was approved by the local institutional (Committee on Ethics of Biomedicine, Second Military Medical University) review board, and written informed consent was waived for each patient.

\section{Consent for publication}

Not applicable

\section{Competing interests}

The authors declare that they have no competing interests

\section{Author details}

${ }^{1}$ Department of Radiology, Changhai Hospital of Shanghai, Shanghai, China.

${ }^{2}$ Huiying Medical Technology Co., Ltd, Beijing, China.

Received: 2 August 2019 Accepted: 29 October 2019

Published online: 12 November 2019

\section{References}

1. Ferlay J, Soerjomataram I, Dikshit R, Eser S, Mathers C, Rebelo M, et al. Cancer incidence and mortality worldwide: sources, methods and major patterns in GLOBOCAN 2012. Int J Cancer. 2015;136:E359-86.

2. Chen W, Zheng R, Baade PD, Zhang S, Zeng H, Bray F, et al. Cancer statistics in China, 2015. CA Cancer J Clin. 2016:66:115-32.

3. Benson AB, Venook AP, Al-Hawary MM, Cederquist L, Chen YJ, Ciombor KK, et al. Rectal Cancer, version 2.2018, NCCN clinical practice guidelines in oncology. J Natl Compr Cancer Netw. 2018;16:874-901.
4. Lee YC, Hsieh CC, Chuang JP. Prognostic significance of partial tumor regression after preoperative chemoradiotherapy for rectal cancer: a metaanalysis. Dis Colon Rectum. 2013;56:1093-101.

5. Gillies RJ, Kinahan PE, Hricak H. Radiomics: images are more than pictures, They Are Data. Radiology. 2016;278:563-77.

6. Lubner MG, Stabo N, Abel EJ, Del Rio AM, Pickhardt PJ. CT textural analysis of large primary renal cell carcinomas: pretreatment tumor heterogeneity correlates with histologic findings and clinical outcomes. AJR Am J Roentgenol. 2016;207:96-105.

7. Sidhu HS, Benigno S, Ganeshan B, Dikaios N, Johnston EW, Allen C, et al. Textural analysis of multiparametric MRI detects transition zone prostate cancer. Eur Radiol. 2017;27:2348-58

8. Vargas HA, Veeraraghavan $\mathrm{H}$, Micco M, Nougaret S, Lakhman Y, Meier AA et al. A novel representation of inter-site tumour heterogeneity from pretreatment computed tomography textures classifies ovarian cancers by clinical outcome. Eur Radiol. 2017;27:3991-4001.

9. Ueno Y, Forghani B, Forghani R, Dohan A, Zeng XZ, Chamming's F, et al. Endometrial carcinoma: MR imaging-based texture model for preoperative risk stratification-a preliminary analysis. Radiology. 2017;284:748-57.

10. Grone J, Loch FN, Taupitz M, Schmidt C, Kreis ME. Accuracy of various lymph node staging criteria in rectal Cancer with magnetic resonance imaging. J Gastrointest Surg. 2018;22:146-53.

11. Balyasnikova S, Read J, Wotherspoon A, Rasheed S, Tekkis P, Tait D, et al Diagnostic accuracy of high-resolution MRI as a method to predict potentially safe endoscopic and surgical planes in patients with early rectal cancer. BMJ Open Gastroenterol. 2017:4:e000151.

12. Sun $Y$, Hu P, Wang J, Shen L, Xia F, Qing G, et al. Radiomic features of pretreatment MRI could identify T stage in patients with rectal cancer: preliminary findings. J Magn Reson Imaging. 2018.

13. Horvat N, Veeraraghavan $\mathrm{H}$, Khan M, Blazic I, Zheng J, Capanu M, et al. MR imaging of rectal Cancer: Radiomics analysis to assess treatment response after Neoadjuvant therapy. Radiology. 2018;287:833-43.

14. Mahul BA, Stephen E, Frederick LG. AJCC cancer staging manual. 8th ed. New York: Springer; 2016.

15. Nougaret S, Reinhold C, Mikhael HW, Rouanet P, Bibeau F, Brown G. The use of MR imaging in treatment planning for patients with rectal carcinoma: have you checked the "DISTANCE"? Radiology. 2013:268:330-44.

16. Cho EY, Kim SH, Yoon JH, Lee Y, Lim YJ, Kim SJ, et al. Apparent diffusion coefficient for discriminating metastatic from non-metastatic lymph nodes in primary rectal cancer. Eur J Radiol. 2013;82:e662-8.

17. Curvo-Semedo L, Lambregts DM, Maas M, Beets GL, Caseiro-Alves F, BeetsTan RG. Diffusion-weighted MRI in rectal cancer: apparent diffusion coefficient as a potential noninvasive marker of tumor aggressiveness. J Magn Reson Imaging. 2012;35:1365-71.

18. Sun Y, Tong T, Cai S, Bi R, Xin C, Gu Y. Apparent diffusion coefficient (ADC) value: a potential imaging biomarker that reflects the biological features of rectal cancer. PLoS One. 2014;9:e109371.

19. Tang $C$, Lin MB, Xu JL, Zhang LH, Zuo XM, Zhang ZS, et al. Are ADC values of readout-segmented echo-planar diffusion-weighted imaging (RESOLVE) correlated with pathological prognostic factors in rectal adenocarcinoma? World J Surg Oncol. 2018;16:138.

20. Al-Sukhni E, Milot L, Fruitman M, Beyene J, Victor JC, Schmocker S, et al. Diagnostic accuracy of MRI for assessment of T category, lymph node metastases, and circumferential resection margin involvement in patients with rectal cancer: a systematic review and meta-analysis. Ann Surg Oncol. 2012;19:2212-23.

21. Dewhurst $C$, Rosen MP, Blake MA, Baker ME, Cash BD, Fidler JL, et al. ACR appropriateness criteria pretreatment staging of colorectal cancer. J Am Coll Radiol. 2012;9:775-81.

22. Tezcan D, Turkvatan A, Turkoglu MA, Bostanci EB, Sakaogulllari Z Preoperative staging of colorectal cancer: accuracy of single portal venous phase multidetector computed tomography. Clin Imaging. 2013;37:1048-53.

23. Bipat S, Glas AS, Slors FJ, Zwinderman AH, Bossuyt PM, Stoker J. Rectal cancer: local staging and assessment of lymph node involvement with endoluminal US, CT, and MR imaging--a meta-analysis. Radiology. 2004;232:773-83.

24. Huang YQ, Liang CH, He L, Tian J, Liang CS, Chen X, et al. Development and validation of a Radiomics Nomogram for preoperative prediction of lymph node metastasis in colorectal Cancer. J Clin Oncol. 2016;34:2157-64.

\section{Publisher's Note}

Springer Nature remains neutral with regard to jurisdictional claims in published maps and institutional affiliations. 